International Journal of Medical Sciences

ISSN 1449-1907 www.medsci.org 2008 5(2):100-105

Research Paper

(C) Ivyspring International Publisher. All rights reserved

\title{
MAINTENANCE HORVONAL TREATMENT IMPROVES PROGRESSION FREE SURVIVAL AFTER A FIRST LINE CHEMOTHERAPY IN PATIENTS WITH METASTATIC BREAST CANCER
}

\section{Armelle Dufresne 1, Xavier Pivot 2, Christophe Tournigand 3 , Thomas Facchini 4, Thierry Alweeg ${ }^{5}$, Loic Chaigneau ${ }^{2}$, Aimery De Gramont ${ }^{3}$}

1. University Hospital E. Herriot, Lyon, France

2. University Hospital J. Minjoz, Besancon, France

3. University Hospital Sain Antoine, Paris, France

4. Cancer Center, Reims, France

5. Cancer Center, Dijon, France

Correspondence to: Professor Xavier Pivot, M.D., Ph.D., University hospital of J. Minjoz, 25000 Besancon France. Xavier.pivot@univ-fcomte.fr

Received: 2008.03.13; Accepted: 2008.05.04; Published: 2008.05.05

The present study was conducted in patients with metastatic breast cancer. Its aim was to identify the factors which influence progression -free survival (PFS) and overall survival (OS) after the first line of chemotherapy in patients with positive tumour hormone receptor status. The patients with early disease progression during first-line chemotherapy were not included. In total, 560 patients who achieved a stable disease or a response to first-line chemotherapy were studied. The factors identified to improve the duration of PFS or OS in multivariate analysis were: number of metastatic sites $(p=.01 ; p=.01)$, metastatic sites $(p=.02 ; p=.04)$, Disease free interval $(\mathrm{p}=.001 ; \mathrm{p}<.0001)$, previous hormonal therapy $(\mathrm{p}=.03 ; \mathrm{p}=\mathrm{ns})$, response to first line chemotherapy $(\mathrm{p}<.0001 ; \mathrm{p}$ $=0.0001)$ and an administration of maintenance hormonal therapy $(\mathrm{p}<.0001 ; \mathrm{p}=.001)$. The major impact obtained by maintenance hormonal treatment after first-line chemotherapy in this study seems to indicate that this strategy should be recommended in patients with an ER or PgR positive tumour.

Key words: Breast cancer; maintenance treatment; chemotherapy; Metastatic; hormonotherapy.

\section{INTRODUCTION}

Breast cancer is the most common cancer among women in the USA and Western Europe. World incidence was 1050000 cases during the year 2000 with a mortality rate of 373000 [1]. Despite earlier diagnosis and improvement in adjuvant therapies some patients will present metastatic recurrence. Then, the disease is incurable and the median of survival is 18 to 24 months [2-3]. The use of systemic therapies such as hormonal therapy, chemotherapy or new biological treatment is to reduce tumour masses, improve survival and preserve quality of life. Whatever the initial efficacy of the treatment undertaken in metastatic setting, almost every patient will relapse. The main goal is to improve progression free survival (PFS). To achieve this, the type of chemotherapy, the optimal duration of chemotherapy, the benefit of maintenance chemotherapy, the benefit of maintenance hormonal treatment are debatable. The present study was conducted to identify the factors which influence progression-free survival after the first line of chemotherapy. Among them, the present study focuses on the impact of hormonal maintenance therapy and constitutes the largest retrospective study on this subject.

\section{PATIENTS AND METHODS}

\section{Study population}

This study included 934 patients treated for metastatic breast cancer in 4 French cancer centres. The diagnosis of metastasis was made between 1992 and 2002. A total of 772 patients received first-line chemotherapy [4]. Because the present analysis focuses on the impact of hormonal treatment beyond first line chemotherapy, we included only patients with positive tumour hormonal receptor status established on the primary tumour. When early disease progression occurs at first chemotherapy response assessment or within 3 months after the first cycle of chemotherapy in metastatic disease, one can consider that it is a failure of chemotherapy. It will not be relevant to search in this subset for factors which influence progression-free survival (PFS). Those cases were excluded from the present analysis. In total, 560 
patients were studied to detect predictive factors to the duration of PFS after first-line chemotherapy and among those factors the impact of hormonal treatment given as maintenance therapy was analysed.

\section{Statistical analysis}

The duration of PFS is defined as the time from the beginning of first line chemotherapy treatment to the date of progressive disease or death. Metastatic survival is defined as the time from the diagnosis of a metastasis to date of death or last follow-up. PFS, metastatic and overall survival were estimated using the Kaplan-Meier method. Comparisons were performed using the log-rank test. Proportional hazards Cox model was used to identify which factors could influence the duration of PFS. Each significant variable in univariate analysis was included in multivariate analysis. The adjusted Hazard Ratio (ad-HR) was provided for each significant variable. The following variables were included in the Cox model: menopausal status (pre- versus postmenopause); nodal involvement of the primary tumour (positive versus negative); hormonal receptor (HR) status (positive if estrogen receptors and/or progesterone receptors are positive versus negative); initial surgery (partial versus radical mastectomy); adjuvant chemotherapy (yes versus no); adjuvant hormonal therapy (yes versus no); complementary radiotherapy (yes versus no); disease free interval (DFI) between the date of diagnosis of breast cancer and the date of first diagnosis of metastatic disease (under or above two years); metastatic site (bone and/or node and/or skin and/or pulmonary versus liver); number of metastatic sites (single versus multiple); type of first-line chemotherapy (anthracycline- and/or taxane-containing regimen versus other); previous line of hormonotherapy administered in metastatic setting before the first-line chemotherapy (no versus yes); best response to first-line chemotherapy (complete (CR) or partial response (PR) versus stable disease (SD) defined according to recist criteria); maintenance hormonal therapy (yes versus no).

\section{RESULTS}

A total of 560 patients were studied and table 1 describes patients' characteristics. Maintenance hormonal therapy was given alone after chemotherapy in 308 patients. The hormonal treatment was tamoxifen (94), aromatase inhibitors (153), fulvestran (47) and megesterol acetate (14). The median duration of first line chemotherapy was 4.4 months (ranges: 3 - 9.7). The factors identified to improve the duration of PFS in multivariate analysis were: number of metastatic sites $(p=.01)$, metastatic sites $(p=.02)$, Disease free interval $(\mathrm{p}=.001)$, previous hormonal therapy $(\mathrm{p}=$ $.03)$, response to first line chemotherapy $(p<.0001)$ and an administration of maintenance hormonal therapy $(\mathrm{p}<.0001)$. The factors related to an increase of OS duration in multivariate analysis were: number of metastatic sites $(p=.01)$, metastatic sites $(p=.04)$, Disease free interval $(p<.0001)$, response to first line chemotherapy $(p=0.0001)$ and an administration of maintenance hormonal therapy $(p=.001)$.

Table 1. Patients characteristics.

\begin{tabular}{|c|c|c|}
\hline Population & $\begin{array}{l}\text { Number } \\
560\end{array}$ & $\%$ \\
\hline \multicolumn{3}{|l|}{ Menopausal status } \\
\hline Post menopause & 321 & 57 \\
\hline Pre menopause & 239 & 43 \\
\hline \multicolumn{3}{|l|}{ Node involvement } \\
\hline Negative & 152 & 27 \\
\hline Positive & 257 & 46 \\
\hline Unknown & 151 & 27 \\
\hline \multicolumn{3}{|l|}{ Initial surgery } \\
\hline Total Mastectomy & 230 & 41 \\
\hline Partial Mastectomy & 198 & 35 \\
\hline Not done & 132 & 24 \\
\hline \multicolumn{3}{|l|}{ Radiotherapy } \\
\hline Yes & 353 & 63 \\
\hline No & 59 & 11 \\
\hline Unknown & 148 & 26 \\
\hline \multicolumn{3}{|l|}{ Adjuvant Chemotherapy } \\
\hline Yes & 244 & 44 \\
\hline No & 166 & 29 \\
\hline Unknown & 150 & 27 \\
\hline \multicolumn{3}{|l|}{ Adjuvant hormonotherapy (tamoxifen) } \\
\hline Yes & 202 & 36 \\
\hline No & 187 & 33 \\
\hline Unknown & 171 & 31 \\
\hline \multicolumn{3}{|l|}{ Disease free interval } \\
\hline$<2$ years & 109 & 19 \\
\hline$\geq 2$ years & 318 & 57 \\
\hline \multicolumn{3}{|c|}{ Previous line of hormonotherapy in metastatic setting } \\
\hline Yes & 173 & 31 \\
\hline No & 387 & 69 \\
\hline \multicolumn{3}{|l|}{ Number of metastatic sites } \\
\hline Single & 311 & 56 \\
\hline Multiple & 249 & 44 \\
\hline \multicolumn{3}{|l|}{ Metastatic sites } \\
\hline Liver involvement & 194 & 35 \\
\hline Others & 366 & 65 \\
\hline \multicolumn{3}{|l|}{ First line chemotherapy } \\
\hline Anthracyclines and/or taxanes & 401 & 72 \\
\hline Others & 159 & 28 \\
\hline \multicolumn{3}{|l|}{ Best response to chemotherapy } \\
\hline SD & 180 & 32 \\
\hline $\mathrm{RC}$ or $\mathrm{RP}$ & 324 & 58 \\
\hline Unknown & 56 & 10 \\
\hline \multicolumn{3}{|l|}{ Maintenance hormonal therapy } \\
\hline Yes & 308 & 55 \\
\hline No & 252 & 45 \\
\hline
\end{tabular}


Tables 2 and 3 list the significant predictive factors for the duration of PFS and OS after first-line chemotherapy. Figures 1 and 2 show the patients' PFS and OS from the first line of chemotherapy according to maintenance hormonal status.

Table 2. Significant predictive factors for PFS duration after a first line chemotherapy.

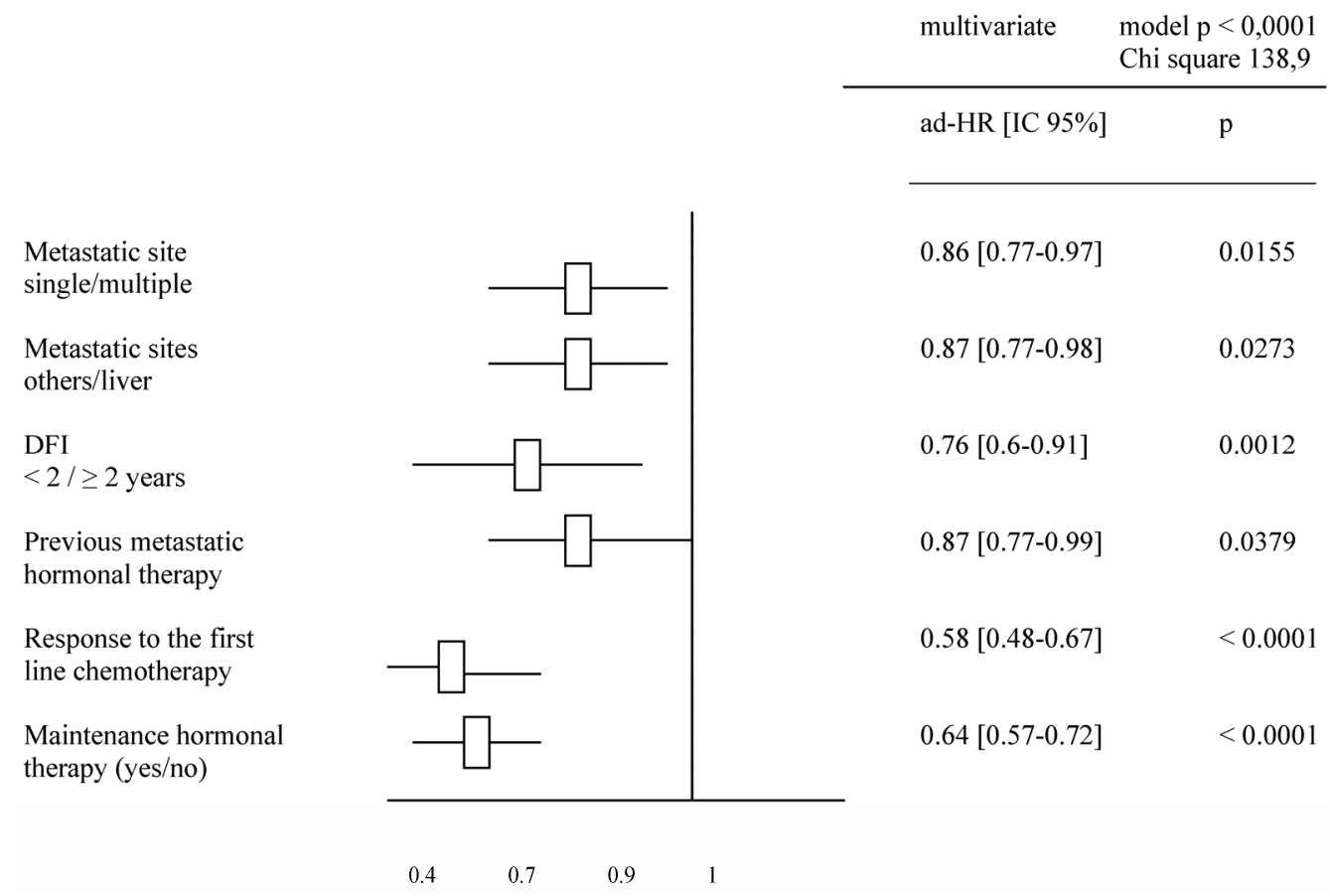

Table 3. Predictive factors for OS duration after a first line chemotherapy.

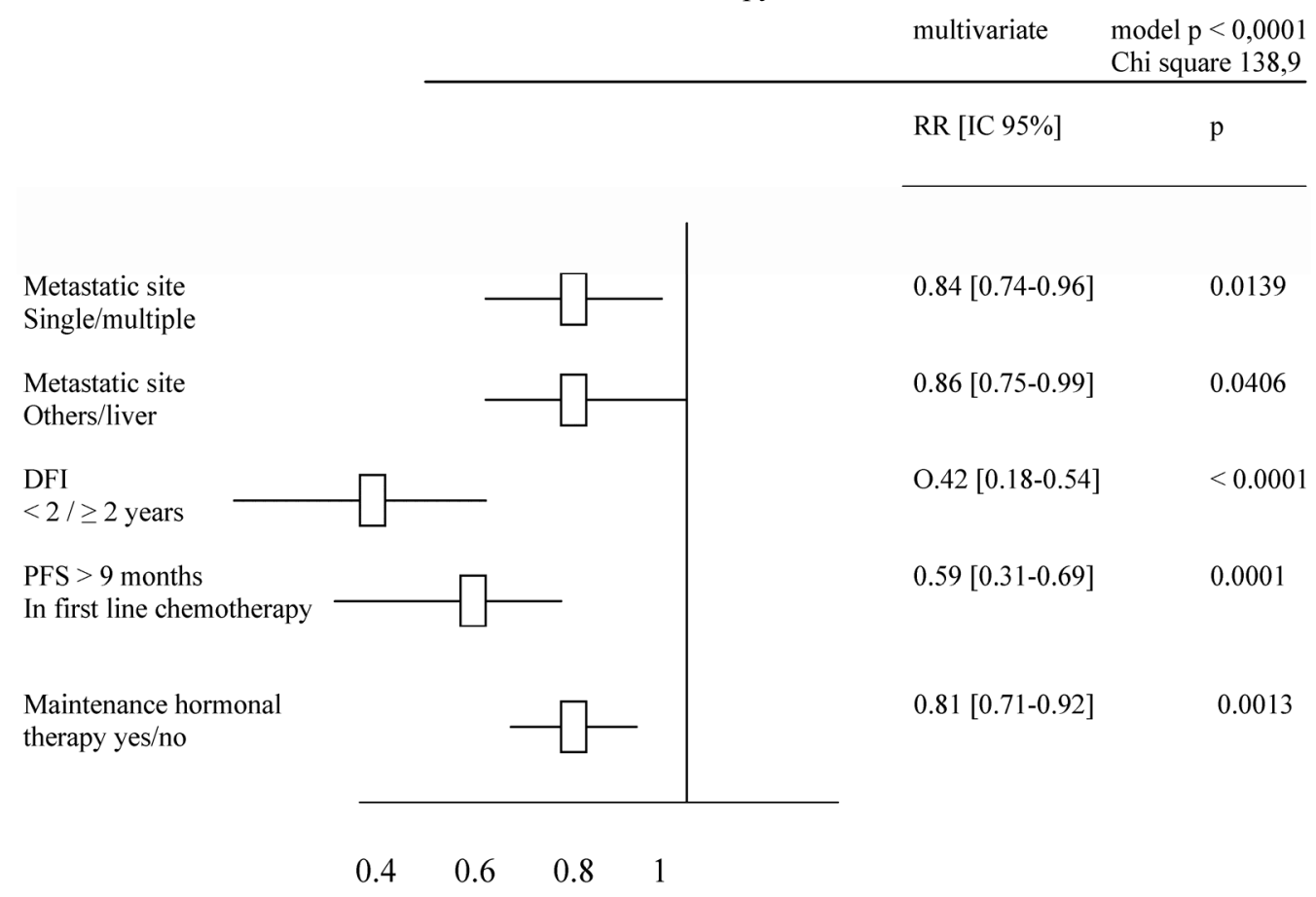




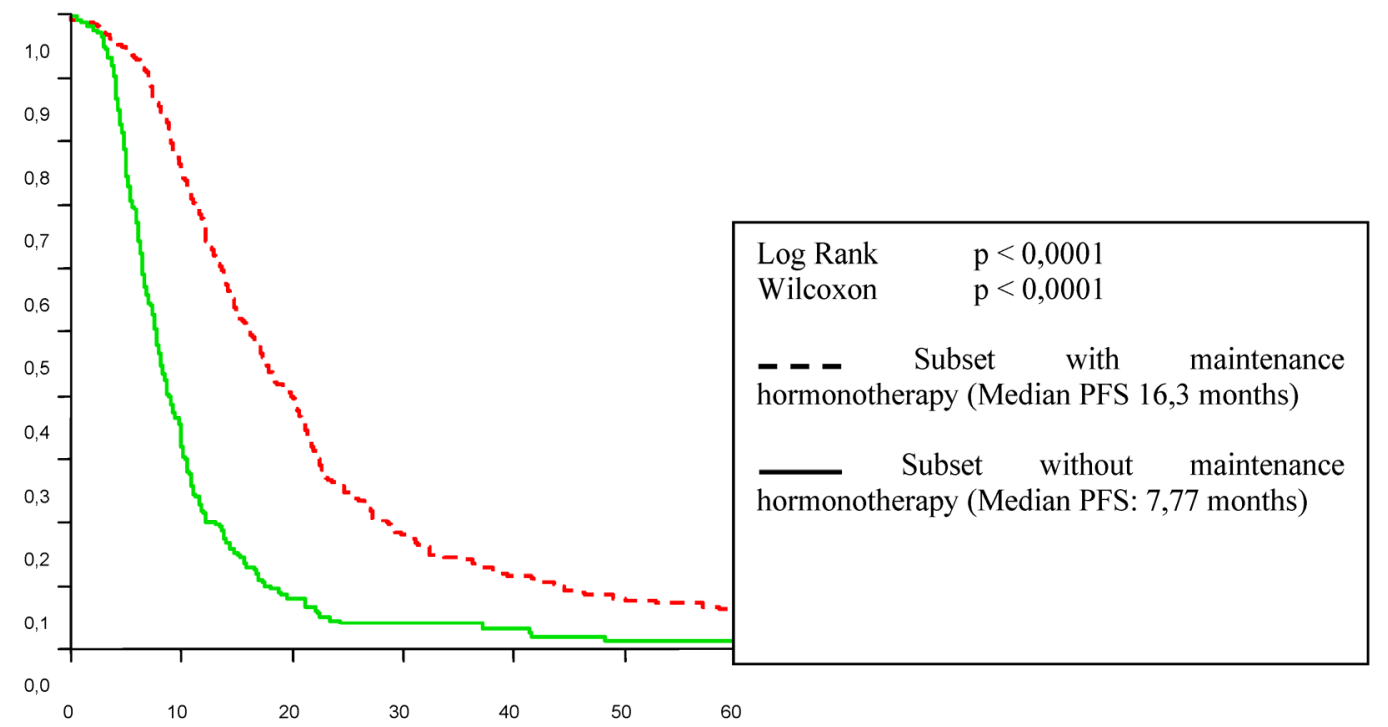

Figure 1. Progression free survival in first-line chemotherapy according to maintenance hormonal treatment status.

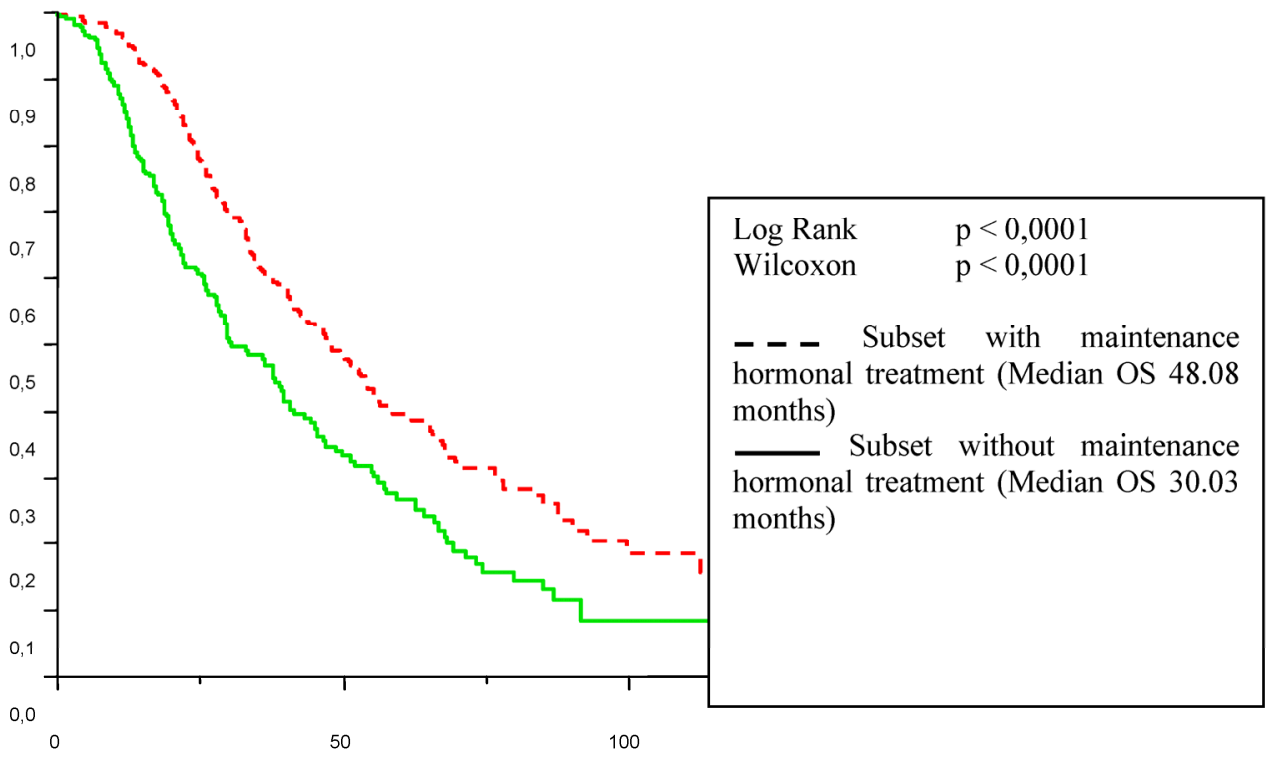

Figure 2. Patient overall survival (OS) from the first line chemotherapy according to maintenance hormonal treatment status.

\section{DISCUSSION}

The search for prognostic and predictive factors that could influence the survival of patients treated for metastatic breast cancer has already been the subject of several studies. It seems that 2 components in the natural outcome of tumours must be considered. The first category is related to the primary characteristics such as initial histological grade, hormonal receptor status. The second category is linked to the metastatic characteristics: proliferation index reflected by the length of disease-free interval, type and number of metastatic sites involved. On the other hand, some prognostic factors are linked to the treatments undertaken, stressing their impact on the natural outcome of the disease: type of hormonotherapy, type of chemotherapy, type of response achieved by treatment [5-12]. The impact of some factors remains debatable, such the duration of treatment. The optimal duration of chemotherapy in patients who respond or have stable disease is not identified. In 1987, Coates compared continuous chemotherapy (until progression or toxicity) versus intermittent chemotherapy (stop after three cycles and re-treatment at the time of disease progression) [13]. Patients receiving continuous therapy had superior response rates, time to progression, and quality-of-life scores, but no improvement in survival was observed. A 
similar trial conducted by the Piedmont Oncology Association randomly assigned patients who had responding or stable disease after six cycles of CAF to either CMF or observation. In the observation subset, CMF was given when disease progression occurred [14]. Time to progression was three times longer in patients under continuous therapy than for those with interrupted treatment $(9.4$ vs. 3.2 months, respectively), but overall survival in both groups was similar. Falkson et al randomly assigned 141 patients whose measurable disease showed a complete response after six cycles of CAF to receive either maintenance chemo-hormonal therapy or observation [15]. Time to disease progression was 19 months in patients who received the maintenance treatment versus 8 months in patients under observation but again the overall survival curves were similar in both groups. The French Epirubicin Study Group study, Gregory trial and Nooij study lead to the same results when they compared interrupted with prolonged chemotherapy regimen: continuous therapy tends to improve duration of response and progression-free survival without a significative impact on overall survival [16-18]. In total, a chemotherapy holiday is associated with a shorter time-to-progression but no adverse effect on survival. While in some studies, continuous chemotherapy seemed not to affect the quality of life [13, 18], several studies showed increased rates of adverse effects [14, 15, 17]. Definitively, the major limit to the use of prolonged regimens of chemotherapy is related to their toxicity, all the more so as they are cumulative (cardiac toxicity of anthracyclins, neurologic toxicity of taxanes, haematological cumulative toxicities with any chemotherapy...). The proposition to give hormonal treatment to prolong therapy in hormonal-positive tumors is another possible option. In the literature, data focused on this strategy are rare. Only one prospective randomised study published by Kloke et al in 1999 is available [19]. In this phase-III trial, 90 patients with a disease controlled after 6 cycles of anthracyclin- and ifosfamide-containing regimen were randomised to receive or not maintenance therapy by medroxyprogesterone acetate. A longer median time-to-progression was reported among patients who were treated by maintenance hormonotherapy (4. 9 versus 3.7 months; $p=0.02$ ). Two retrospective studies found hormonal maintenance therapy as a significant factor among several prognostic factors for disease-free survival and overall survival after first line chemotherapy. In 1997, Berruti et al analysed the factors influencing response rate and overall survival among 207 patients treated by epirubicin, followed or not by maintenance hormonotherapy [20]. The patients who received maintenance hormonotherapy survived significantly longer than those submitted to observation in uni- and multivariate analysis. The author concluded that "the positive impact of maintenance hormonal therapy is impressive and deserves confirmation in randomized studies". Montemurro et al studied 109 patients receiving high-dose chemotherapy and analysed the factors which improve its efficacy [21]. Maintenance hormonal therapy appeared to be a significant factor in multivariate analysis. The maintenance hormonal treatment improved the progression-free survival from $19,2$ to 31,1 months ( $p=0,022)$.

The influence of the type of response achieved by first line chemotherapy is well established [11]. Strikingly, in the present study, hormonal treatment administered after response or stabilisation with first-line chemotherapy seemed related to a better outcome with 7.8 to 16.3 months for the duration of PFS ( $p<0.0001)$ and from 30 to 48.1 months for the overall duration of metastatic survival $(p<0$. 0001). This benefit was observed independently of the type of response achieved by first line chemotherapy. One can object that the choice of patient/tumour characteristics for who would or would not receive the maintenance hormonal therapy was not random, or controlled in any way. This may have led to a selection of better prognosis patients. We cannot know whether we are observing natural history or impacting it in such a trial.

Nevertheless the major impact obtained by maintenance hormonal treatment after the first line chemotherapy might indicate that this strategy should be recommended in patients with an ER or PgR positive tumour. Based on the amplitude of the benefit observed, it may be ethically debatable to conduct a prospective randomized study. Moreover, randomized trials which assess the benefit of a new chemotherapy regimen should allow the possibility to give maintenance hormonal treatment.

\section{Conflict of interest}

The authors have declared that no conflict of interest exists.

\section{References}

1. Remontet L, Esteve J, Bouvier AM et al. Cancer incidence and mortality in France over the period 1978-2000. Rev Epidemiol Sante Publique 2003;51(1 Pt 1):3-30

2. Dickson R, Pestell R, Lippman M. Metastatic breast cancer. In: DeVita T, Hellman S, Rosenberg S, eds. Cancer Principles and practice of oncology, 7 ed. Philadelphia: Lippincot company. 2005:1453-62

3. Ellis M, Hayes D, Lippman M. Treatment of metastatic breast cancer. In: Harris J, Lippman M, Morrow M, Osborne C, eds. Diseases of the breast, 2 ed. Philadelphia: Lippincot company; 2000:749-97.

4. Dufresne A, Pivot X, Tournigand C, Facchni T, Alweeegg T, 
Chaigneau L, De Gramon A. Impact of chemotherapy beyond the fisrt line in patients with metastatic breast cancer. Breast Cancer Res Treat. 2008; 107: 275-279

5. Clark G, Sledge G, Osborne C et al. Survival from first recurrence: relative importance of prognostic factors in 1,015 breast cancer patients. J Clin Oncol 1987; 5: 55-61

6. Rizzieri DA, Vredenburgh JJ, Jones R, et al. Prognostic and predictive factors for patients with metastatic breast cancer undergoing aggressive induction therapy followed by high-dose chemotherapy with autologous stem-cell support. J Clin Oncol 1999; 17(10): 3064-74

7. Nisman B, Barak V, Hubert A, et al. Prognostic factors for survival in metastatic breast cancer during first-line paclitaxel chemotherapy. Anticancer Res 2003; 23(2C): 1939-42

8. Nomura Y. Different survival determinants of metastatic breast cancer patients treated with endocrine therapy or chemo-endocrine therapy. Int J Oncol 1998; 12(4): 817-24

9. Falkson G, Holcroft C, Gelman RS et al. Ten-year follow-up study of premenopausal women with metastatic breast cancer: an Eastern Cooperative Oncology Group study. J Clin Oncol 1995; 13(6): 1453-8

10. Hortobagyi GN, Smith TL, Legha SS, Swenerton KD, Gehan EA, Tap HY, Buzdar AU, Blumenschei GR. Multivariate analysis of prognostic factors in metasttaic breast cancer. J Clin Oncol 1983; 1(12): 776-86

11. Greenberg PA, Hortobagyi GN, Smith TL et al. Long-term follow-up of patients with complete remission following combination chemotherapy for metastatic breast cancer. J Clin Oncol 1996;14(8):2197-205.

12. Pivot X, Asmar L, Buzdar A, Valero V, Hortobagyi GN. An unified definition of clinical anthracycline resistance breast cancer. Br J Cancer 2000; 82: 529-34

13. Coates A, Gebsk V, Bishop JF, et al. Improving the quality of life during chemotherapy for advanced breast cancer. A comparison of intermittent and continuous treatment strategies. N Engl J Med 1987; 317: 1490-1495

14. Muss HB, Case LD, Richards $F$ et al. Interrupted versus continuous chemotherapy in patients with metastatic breast cancer. The Piedmont Oncology Association. N Engl J Med 1991; 325: $1342-1348$

15. Falkson G, Gelman R, Pandya K et al. Eastern Cooperative Oncology Group randomized trials of observation versus maintenance therapy for patients with metastatic breast cancer in complete remission following induction treatment. J Clin Oncol 1998; 16 (5): 1669-1676

16. French Epirubicinn Study Group. Epirubicin-based chemotherapy in metastatic breast cancer patients: role of dose-intensity and duration of treatment. J Clin Oncol 2000; 18 (17): 3115-3124

17. Gregory RK, Powles TJ, Chang JC et al. A randomised trial of six versus twelve courses of chemotherapy in metastatic carcinoma of the breast. Eur J Cancer 1997; 33 (13): 2194-2197

18. Nooi J M, de Haes J, Beex L et al. Continuing chemotherapy or not after the induction treatement in advanced breast cancer patients: clinical outcomes and oncologists' preferences. Eur J Cancer 2003; 39: 614-621

19. Kloke O, Klaasen U, Oberhoff $\mathrm{C}$ et al. Maintenance treatment with medroxyprogesterone acetate in patients with advanced breast cancer responding to chemotherapy: results of a randomized trial. Essen Breast Cancer Study Group. Breast Cancer Res Treat 1999; 55 (1): 51-59

20. Berruti A, Zola P, Buniva T et al. Prognostic factors in metastatic breast cancer patients obtaining objective response or disease stabilization after first-line chemotherapy with epirubicin. Evidence for a positive effect of maintenance hormonal therapy on overall survival. Anticancer Res 1997; 17 (4A): 2763-2768

21. Montemurro F, Rondon G, Ueno NT et al. Factors affecting progression-free survival in hormone-dependent metastatic breast cancer patients receiving high-dose chemotherapy and hematopoietic progenitor cell transplantation: role of maintenance endocrine therapy. Bone Marrow Transplant 2002; 29 (10): 861-866 